\title{
Recognizing Exponential Inter-Contact Time in VANETs
}

\author{
Hongzi Zhu* ${ }^{* \dagger}$ Luoyi Fu*, Guangtao Xue*, Yanmin $\mathrm{Zhu}^{*}$, Minglu Li* and Lionel M. Ni*† \\ * Shanghai Jiao Tong University \\ ${ }^{\dagger}$ Hong Kong University of Science and Technology \\ \{hongzi, yiluofu,gt_xue,yzhu, mlli\}@sjtu.edu.cn,ni@cse.ust.hk
}

\begin{abstract}
Inter-contact time between moving vehicles is one of the key metrics in vehicular ad hoc networks (VANETs) and central to forwarding algorithms and the end-to-end delay. Due to prohibitive costs, little work has conducted experimental study on inter-contact time in urban vehicular environments. In this paper, we carry out an extensive experiment involving thousands of operational taxies in Shanghai city. Studying the taxi trace data on the frequency and duration of transfer opportunities between taxies, we observe that the tail distribution of the intercontact time, that is the time gap separating two contacts of the same pair of taxies, exhibits a light tail such as one of an exponential distribution, over a large range of timescale. This observation is in sharp contrast to recent empirical data studies based on human mobility, in which the distribution of the inter-contact time obeys a power law. By performing a least squares fit, we establish an exponential model that can accurately depict the tail behavior of the inter-contact time in VANETs. Our results thus provide fundamental guidelines on design of new vehicular mobility models in urban scenarios, new data forwarding protocols and their performance analysis.
\end{abstract}

Index Terms-vehicular ad-hoc networks; inter-contact time distribution; exponential tail; empirical data analysis

\section{INTRODUCTION}

Vehicular ad hoc networks (VANETs) are recognized as an important component in the next generation of intelligent transportation systems, to improve safety, security and efficiency of transportation systems and enable new mobile services to the public. In VANETs, vehicles equipped with wireless communication devices can transfer data with each other (vehicle-to-vehicle communications) as well as with the roadside infrastructure (vehicle-to-roadside communications). In order to successfully transfer data from a vehicle to another, the vehicle needs to first wait until it geographically meets other vehicles (within the communication range of each other) for data-relay. Applications based on this type of data transfer will strongly depend on vehicular mobility characteristics, especially on how often such communication opportunities take place and on how long they last.In this paper, we focus on studying the metric called inter-contact time [1], [13], [14], which denotes the time elapsed between two successive contacts of the same two vehicles. Since data transfer arises in a store-carry-forward fashion, the inter-contact time of the two vehicles is a major component of the end-to-end delay, as it presents how long it takes to encounter the other mobile vehicle to have any chances to forward/relay the data for communications. Larger inter-contact time results in larger end-to-end delay.

In the literature, there have been many studies on the characteristics of the inter-contact time in delay tolerant networks (DTNs) and mobile ad hoc networks (MANETs). Most of these results focus on theoretical models such as random walk mobility models (RWM) [2]-[4], random waypoint mobility models (RWP) [6]-[8] and random direction mobility models (RDM) [9]. For example, numerical simulations [4], [10], [11] based on RWP models show that the inter-contact time is exponentially distributed. Further, authors in [12] rigorously prove that a finite domain is one of the key aspects in creating the exponential inter-contact time tail distribution. This is because finite boundaries actually force mobile node to move only within a certain region and hence increase the meeting opportunities between nodes. While theoretical mobility models facilitate problem analysis, they are far beyond reality and not practical in designing networking protocols for real systems and their performance analysis. Recently, some empirical results [1], [13], [14] based on human mobility show that the tail distribution of the inter-contact time is far from being exponential, but can be approximated or lower bounded by a power law. In order to have a better understanding of practical constraints in opportunistic data transfer between vehicles, experiments involving thousands of vehicles over a long time span of months are in pressing demand. However, due to vast deployment costs, there is no existing work, to the best of our knowledge, studying vehicular inter-contact time distribution in urban settings based on real experiments.

In this paper, we collect real motion traces from about 2,100 operational taxies for over one month in Shanghai city, the biggest metropolis in China. We then check contacts between each pair of taxies using a sliding time window. By analyzing the large volume of trace data, we surprisingly find that the tail distribution of the inter-contact time between taxies follows an exponential distribution on a large range of timescale, other than a power law exhibited in human mobility [1], [13], [14]. This implies that, to some extent, taxies can frequently meet with each other. Thus, data delivery would experience smaller end-to-end delay. We further perform a least squares fit to establish the accurate model capturing the tail behavior of the inter-contact time in VANETs. Our findings thus provide fundamental guidelines on design of new vehicular mobility models in urban scenarios, new data forwarding protocols and their performance analysis. 


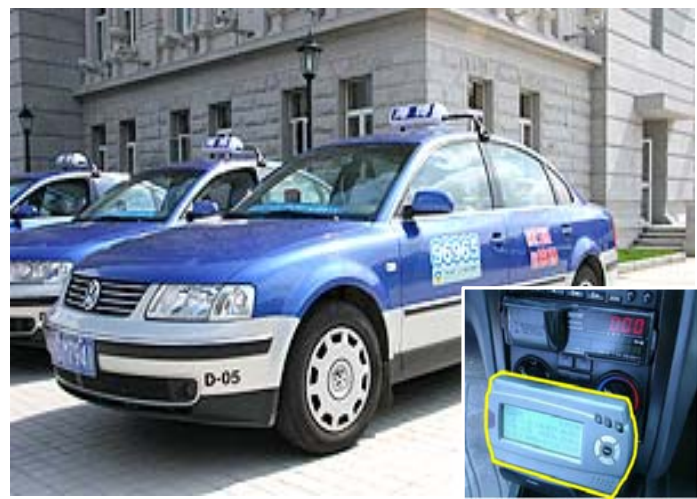

Fig. 1. A taxi with a commercial GPS device installed, the highlight area in the inset shows a device consisting of a commercial GPS receiver and a GPRS wireless communication module.

The rest of this paper is structured as follows. Section II is dedicated to related work. We describe the empirical data analysis in Section III. In Section IV, we present the exponential model that can perfectly depict the tail behavior of the inter-contact time. Finally, concluding remarks and outline the directions for future work are described in Section V.

\section{RELATED WORK}

In VANETs, to perform data transfer, two vehicles have to wait until they are within each other's communication range. This type of data transfer paradigm is much related to both delay-tolerant networking and mobile ad hoc networking. In the literature, bunches of studies have made their effort on revealing the relationship between the underlying mobility models of nodes and the consequent characteristics of the inter-contact time in MANETs. In general, these studies can be classified into two categories: theoretical mobility models based and empirical trace based.

A majority of research results have uncovered a common property of many theoretical mobility models that the tail of the inter-contact distribution decays exponentially. In other words, for these models, the inter-contact time is light tailed. For example, authors in [4], [10], [11] draw this conclusion through numerical simulations based on RWP mobility models. Furthermore, some theoretical results show that the first and second moments of the inter-contact time are bounded above under Brownian motion model on a sphere. In particular, authors in [12] prove that a finite boundary is a major factor that causes the exponential tail behavior under any RWP mobility model and any RWM mobility model. While using theoretical mobility models simplifies problem analysis, they are inconsistent with the reality and thus impractical in designing networking protocols for real systems.

In recent years, there has emerged more research work taking experimental study on the characteristics of the intercontact time. For example, authors in [1], [13], [14] find that the tail behavior of the inter-contact time based on human mobility is far from being exponential but is close to a power law

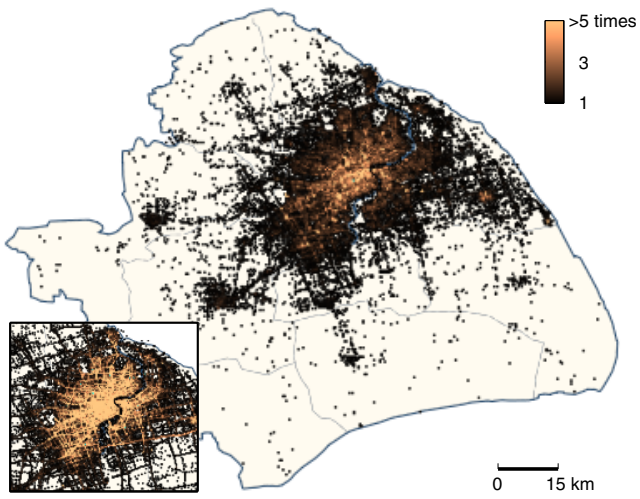

Fig. 2. The geometry of destinations of all taxi deliveries on Shanghai map during February of 2007. Every colored dot presents the average number of destinations per taxi per day located in a corresponding $300 \mathrm{~m} \times 300 \mathrm{~m}$ square area on the map. The inset shows aggregated GPS report distribution (destinations not involved).

instead. These results are based on real traces such as human contacts while at conferences [14], campus WiFi login records [13], [15] and a Bluetooth network containing hundreds of people in an office [14]. It is apparent that the mobility of vehicles is significantly different from that of human beings in terms of speed, constraints of road transportation systems and travel distance. Although these empirical results based on human mobility depict another scene of the inter-contact time distribution, the situation in vehicular environments is still left unknown.

DieselNet [16] at UMass consisting of 40 buses studies the aggregated inter-contact time distribution at a granularity of bus route and find a clear periodic structure in the inter-contact times between two bus routes. For the lack of enough contact samples between two individual buses, the bus trace data are not sufficient for studying the distribution of the inter-contact time between two individual buses. In the RAPID routing protocol [17], it is assumed that the distribution of bus intercontact times is exponential to make their problem tractable.

\section{EMPIRICAL DATA ANALYSIS}

In order to investigate the frequency and duration of contacts between vehicles in urban environments, it is of great importance to study real trace data involving a large number of mobile vehicles. In this section, we first give a brief description of the taxi trace data collected in the SG project [18], [19]. We then present the inter-contact time characteristics embedded in the trace data. Finally, we discuss the possible reasons behind our key observation on the vehicular inter-contact time distribution.

\section{A. Collecting Vehicular Trace Data}

To study realistic vehicular mobility in urban scenarios, an experiment which involves a large number of vehicles and covers a sufficiently long time period is essential. 


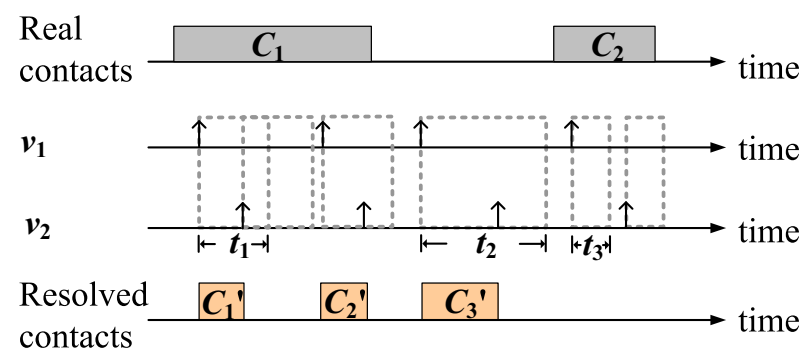

Fig. 3. Extract contacts from GPS reports of vehicle v1 and v2. Boxes in dotted line denote sliding time windows of different granularities used to check contacts. Individual GPS reports are presented by short arrow line segments.

For this purpose, we collect motion trace data from 2,100 taxies in Shanghai city during the whole month of February in 2007 (available at http://www.cse.ust.hk/dcrg). The geometry of destinations of all taxi deliveries on Shanghai map is shown in Fig. 2. A commuting taxi equipped with a commercial GPS receiver and a GPRS wireless communication module is shown in Fig. 1. As a taxi runs along the roads in the city, it periodically sends a report back to a data center via a GPRS channel. Due to the GPRS communication cost for data transmission, reports are sent at a time interval of one minute reports are sent at a time interval of one minute when a taxi is loaded and of about 15 seconds when it is vacant. The information contained in such a report includes: the taxi's ID, the longitude and latitude coordinates of the taxi's current location, report timestamp, the instant speed and heading angle of the taxi and the status of the taxi (i.e., whether the taxi has passengers onboard).

\section{B. Computing Inter-Contact Times}

In this paper, we are interested in how often transfer opportunities can occur between vehicle pairs since it is the key factor that impacts the end-to-end delay for data delivery in VANETs.

1) Contact Extraction from Trace Data: Ideally, all connection opportunities encountered twenty-four hours a day, with a granularity measured in seconds should be recorded in the data for study. Since we collect GPS reports in discrete time, we use a sliding time window to check contacts between a pair of taxies. Here we make the assumption that two vehicles would be able to communicate (called a contact) if their locations reported within the time window are within the communication range. For the example in Fig. 3, suppose we have two real contacts $C_{1}$ and $C_{2}$ happening between vehicle $v_{1}$ and $v_{2}$. As $v_{1}$ and $v_{2}$ keep sending reports, given a communication range, we can slide a time window along the time axis to check contacts.

The above assumption, though, can introduce inaccuracies in the following two cases.

First, if a relatively large time window is used, we may introduce false contacts into consideration. This is because two taxies may have already run far away from their reported locations. Therefore, the retrieved contact may never really exist. For example, in Fig. 3, we may get a false contact $C_{3}^{\prime}$ if a large time window $t_{2}$ is used even though there is no real contact at all. The consequence of introducing false contacts is that it increases the weight of small values of inter-contact times in the distribution since these false contacts cut large inter-contact times into small pieces.

Second, if a small time window is used, we may omit real connection opportunities. This is because two taxies might indeed have a contact but did not send out reports simultaneously. In this case, we may not capture this contact due to the small size of the time window. This is the case of contact $C_{2}$ as shown in Fig. 3. The consequence of omitting real contacts is that it causes large values of inter-contact times since two real small inter-contact times are now considered as a single huge one. Moreover, using small time windows to check contacts can add the weight of small values into the inter-contact times distribution. For example, in Fig. 3, we will get $C_{1}^{\prime}$ and $C_{2}^{\prime}$ rather than the real one, $C_{1}$, when we take $t_{1}$ to check contacts. To eliminate this effect of using a small time window, we calculate the correlation between two contacts with a small inter-contact time. Specifically, given the reported locations and speeds of each taxi, we calculate the remaining contact time of the first contact as the time these two taxies move along the same directions and at the same speeds before they are out of the communication range. If the second contact is contained within the remaining contact time of the first contact, we make a decision that these two contacts should be merged into one. Vice versa, we can infer when the second contact started and further check whether the first contact can be merged.

Despite these inaccuracies, the taxi GPS trace data are very valuable to study vehicular mobility models since they cover thousands of vehicles and last for one month. In addition, as most of the GPS reports are sent at a relatively small period (48 seconds on average), the deviation of the computed distance of two reported locations within such a small time window from the actual distance between two taxies is small.

2) Inter-Contact Time Computation: We refer to intercontact time as the time elapsed between two successive contacts of the same vehicles as defined in [1], [13], [14]. Specifically, the inter-contact time is computed at the end of each contact, as the time period between the end of this contact and the start of the next contact between the same two vehicles. It should be noted we do not take into consideration the intercontact starting after the last contacts.

\section{Modeling Inter-Contact Time}

The distribution of inter-contact times is computed among all pairs of 2,109 taxies during the whole February in 2007. We get six different sets of inter-contact times by combining different communication ranges and time window sizes used in the contact extraction. The time windows are set to one second, thirty seconds and one minute, respectively, accompanied with two communication ranges of 50 meters and 100 meters [20]. We plot the inter-contact time distribution for the selected trace data in Fig. 4. 


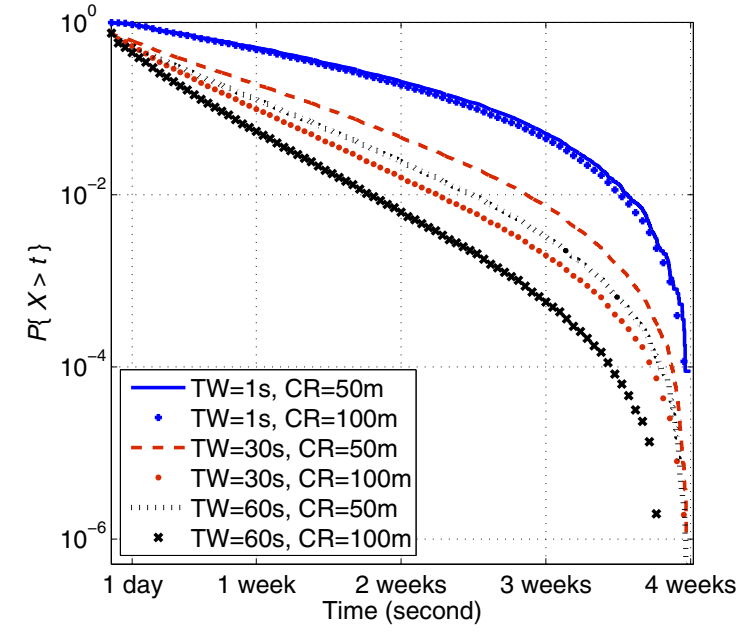

Fig. 4. Tail distribution of the inter-contact time : data collected from 2,109 taxies in Shanghai city during the whole February of 2007. Contacts are collected under three different time windows of one second, thirty seconds and one minute and two communication ranges of 50 meters and 100 meters.

\section{A. Identifying Exponential Inter-Contact Time Tail}

All plots in Fig. 4 describe the tail distribution function, i.e., $P\{X>t\}$, in linear-log scale. The most interesting part in Fig. 4 is that all plots exhibit a very clear exponential tail, i.e., $P\{X>t\} \sim e^{-\beta t}$. This can be indicated by the fact that all plots are almost straight lines with different negative slopes in linear-log scale, from the very beginning of time and over a large range of timescale.

Besides the exponential parts, we also notice that, gradually, all six distributions start to deviate from the exponential decay and drop faster till the end. This rapid cutoff is caused by the limited duration of the trace data, i.e., one month in our experiment. The reason is that inter-contact times that last longer than the duration of the trace data cannot be observed and those ones with very large values close to the duration are less likely to be found. Consequently, the weight of large values of inter-contact times in the distribution is biased. It should be noted that the cutoff part of data should not be used for regression as they can be considered as artifacts to the tail distribution. The effect of limited observation duration has also been noted in Augustin Chaintreau et al's study based on human mobility [1].

\section{B. Establishing Model Parameters}

To identify the exponent constant $\beta$, we perform the leastsquare regression analysis to the extracted inter-contact time. More specifically, we separate the whole regression processing into two steps.

First, we need to identify the divide point from which the tail distribution function stops exponential decay. This can be achieved by seeking for the divide point from which the second derivatives (decay acceleration) of the log-scaled $P\{X>t\}$ are nonzero. The physical meaning of using the second derivatives is clear since a nonzero second derivatives

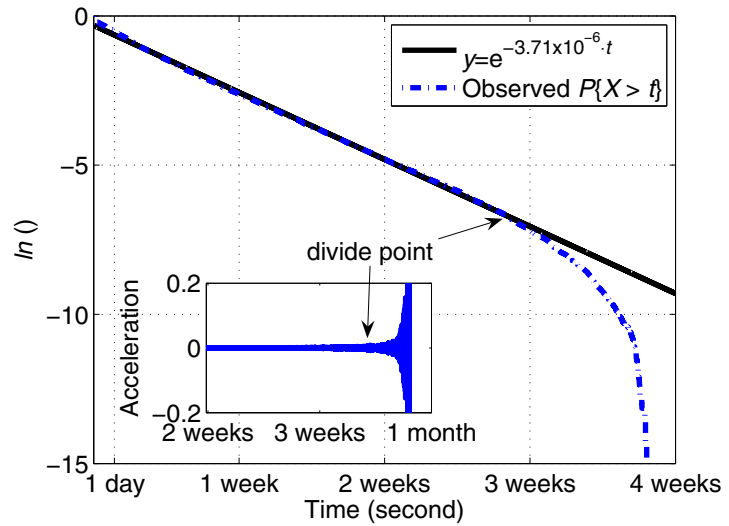

Fig. 5. Tail distribution of the inter-contact time under the time window of one minute and the communication range of 100 meters is very well approximated by an exponential-like distribution $y=\mathrm{e}-? \mathrm{t}$ with $=3.7110$ 6. We omit artifact part in the observed data by examining the nonzero decay acceleration in the tail distribution plot.

of a plot indicates the plot is nonlinear. In practice, we take a small positive value $\varepsilon=0.1$ to determine the divide point.

Second, we apply polynomial regression to the log-scaled $P\{X>t\}$ over the range from the first point to the divide point. The significance of the regression is measured by the coefficient of determination $r^{2}=1-\frac{\sum_{i}\left(y_{i}-\bar{y}\right)^{2}}{\sum_{i}\left(y_{i}-m_{i}\right)^{2}}$, where $y_{i}$ denotes the sample value with mean $\bar{y}$ whereas $m_{i}$ is the modeled value.

For example, we apply this exercise to the lowest plot in Fig. 4. Fig. 5 shows the regression result where the tail distribution of inter-contact time is very well approximated $\left(r^{2}>0.98\right)$ by an exponential distribution $P\{X>t\}=e^{-3.71 \times 10^{-6} t}$ when time is counted in seconds.

The surprising finding of the exponential decay on the inter-contact time tail distribution is thus in a sharp contrast to several recent empirical results on the inter-contact time based on extensive human mobility traces [1], [13], [14]. These results indicate that the tail behavior of the inter-contact time can be approximated or lower bounded by a power law, i.e., $P\{X>t\} \sim t^{-\alpha}$, for some constant $\alpha>0$. More spectacularly, it was shown that the power law exponent $\alpha$ is normally less than one, making the expected end-to-end delay tend to be infinite, independent of any forwarding algorithm, if the network only contains a finite number of devices. In this paper, we claim that the tail distribution of the inter-contact time based on vehicular mobility satisfies an exponential decay or a light tail. An exponential decay means the tail distribution function decreases rapidly over this range. For example, in the lowest plot in Fig. 4, about $45 \%$ of inter-contact times are greater than one day, and only $5 \%$ are greater than one week. This discrepancy calls for the design of new vehicular mobility models in urban scenarios, new data forwarding protocols and their performance analysis. 


\section{CONCLusion AND Future Work}

In this paper we collected real GPS trace data of about two thousand operational taxies for one month. We checked possible contacts between each pair of taxies upon discrete location information. By analyzing all contacts between any pair of taxies, we surprisingly found that the tail distribution of the inter-contact time between taxies follows an exponential distribution on a large range of timescale as opposed to a power low distribution as discovered by previous work. Our results thus provide fundamental guidelines on design of new vehicular mobility models in urban scenarios, new data forwarding protocols and their performance analysis in VANETs.

There are still many aspects for us to investigate in the future. For example, we will investigate what are the key factors that generate exponential tail distributions of intercontact time in VANETs. Theoretical models depicting these key factors should also be created and the soundness of these models should be checked with different vehicles and in different environments. Moreover, it is often assumed in the literature that data transfers can be done instantaneously as soon as two vehicles have a chance to meet. It is definitely not the case in reality where link quality shows very high dynamics. The situation is even worse when consider the same problem in vehicular environments because contacts between vehicles are usually quite short due to high moving speeds and limited communication range. Thus, we will investigate the ultimate end-to-end delay since it can be caused not only by inter-contact times but also by retransmissions if the data transfer fails in a contact.

\section{ACKNOWLEDGMENT}

This research was supported in part by China NSFC Grants 60933011, 60933012, 60903190, 60673166, 90612018 and 60970106, the National Basic Research Program of China (973 Program) under Grant No. 2006CB303000, the National Science and Technology Major Project of China under Grant No. 2009ZX03006-001, Grant No. 2009ZX03006-001 from MIIT of China, STCSM Grant No. 05DZ15005 and Intel IT Research Fund.

\section{REFERENCES}

[1] A. Chaintreau, P. Hui, J. Crowcroft, C. Diot, R. Gass, and J. Scott, "Impact of Human Mobility on the Design of Opportunistic Forwarding Algorithms", in proc. IEEE INFOCOM, 2006.

[2] A. Bar-Noy, I. Kessler, and M. Sidi, "Mobile users: To update or not to update?", In Proc. IEEE INFOCOM, 1994.

[3] A. E. Gamal, J. Mammen, B. Prabhakar, and D. Shah, "Throughput-delay trade-off in wireless networks", in Proc. IEEE INFOCOM, 2004.

[4] G. Sharma, and R. Mazumdar, "Scaling Laws for Capacity and Delay in Wireless Ad Hoc Networks with Random Mobility", in proc. IEEE International Conference on Communication (ICC), 2004.

[5] D. Johnson and D. Maltz, "Dynamic source routing in ad hoc wireless networks", in Mobile Computing, pages 153-181. Kluwer Academic Publishers, 1996.

[6] J. Broch, D. Maltz, D. Johnson, Y. Hu, and J. Jetcheva, "Multi-hop wireless ad hoc network routing protocols", in Proc. the ACM/IEEE MOBICOM, 1998.

[7] C. Chiang and M. Gerla, "On-demand multicast in mobile wireless networks", In Proc. IEEE ICNP, 1998.

[8] P. Johansson, T. Larsson, N. Hedman, B. Mielczarek, and M. Degermark, "Routing protocols for mobile ad-hoc networks - a comparative performance analysis", in Proc. ACM/IEEE MOBICOM, 1999.

[9] E. Royer, P.M. Melliar-Smith, and L. Moser, "An analysis of the optimum node density for ad hoc mobile networks", in Proc. ICC, 2001.

[10] R.Groenevelt, P. Nain, and G. Koole, "Message delay in MANET", in Proc. ACM SIGMETRICS, 2004.

[11] G. Sharma, and R. R. Mazumdar, "Delay and Capacity Trade-off in Wireless Ad Hoc Networks with Random Mobility", in ACM/Kluwer Journal on Mobile Networks and Applications (MONET), 2004.

[12] H. Cai and D.Y. Eun, "Crossing Over the Bounded Domain: From Exponential To Power-law Inter-meeting Time in MANET", in Proc. ACM/IEEE MOBICOM, 2007.

[13] T. Henderson, D. Kotz, and I. Abyzov, "The changing usage of a mature campus-wide wireless network", in Proc. ACM/IEEE Mobicom, 2004.

[14] P. Hui, A. Chaintreau, J. Scott, R. Gass, J. Crowcroft, and C. Diot, "Pocket switched networks and the consequences of human mobility in conference environments," in Proc. ACM SIGCOMM first workshop on delay tolerant networking and related topics (WDTN-05), 2005.

[15] M. McNett, and G. M. Voelker, "Access and mobility of wireless pda user", in Tech. rep., Computer Science and Engineering, UC San Diego, 2004.

[16] X. Zhang, J. Kurose, B. N. Levine, D. Towsley, and H. Zhang, "Study of a Bus-based Disruption-Tolerant Network: Mobility Modeling and Impact on Routing", in Proc. ACM/IEEE MOBICOM, 2007.

[17] A. Balasubramanian, B.N. Levine and A. Venkataramani, "DTN Routing as a Resource Allocation Problem", in Proc. ACM SIGCOMM, 2007.

[18] H. Zhu, Y. Zhu, M. Li, and L.M. Li, "HERO: Online Real-time Vehicle Tracking in Shanghai", in Proc. IEEE INFOCOM, 2008.

[19] H. Zhu, Y. Zhu, M. Li, and L.M. Li, "SEER: Metropolitan-scale Traffic Perception Based on Lossy Sensory Data", in Proc. IEEE INFOCOM 2009.

[20] Y. Gunter, H.P. Gro?mann, " Usage of Wireless LAN for Inter-Vehicle Communication", in Proc. the 8th International IEEE Conference on Intelligent Transportation Systems, 2005. 